Public Health

\section{Hygiene: What and why?}

M ental hygiene, industrial hygiene, oral hygiene, vocal hygiene, respiratory hygiene... There are many "hygienes," but what does the word actually mean? Its definition - the science of preventive medicine and the preservation of health - is broad enough to incorporate concepts such as exercise and diet. But the original and still generally understood usage is in the context of preventing the transmission of infection. Public concerns about SARS (severe acute respiratory syndrome) and current efforts to identify community practices to respond to avian influenza outbreaks or pandemic influenza have returned hygiene concepts to the public sphere. Home hygiene and community hygiene are now recurrent themes in public health messages.

\section{What are hygiene practices?}

7 Hand hygiene, household cleaning and food safety constitute the main focus 迹 for hygiene interventions in the home and community (Box I). Consistent recommendations are to clean hands often by washing with soap and warm water, or to use alcohol-based gel sanitizers if

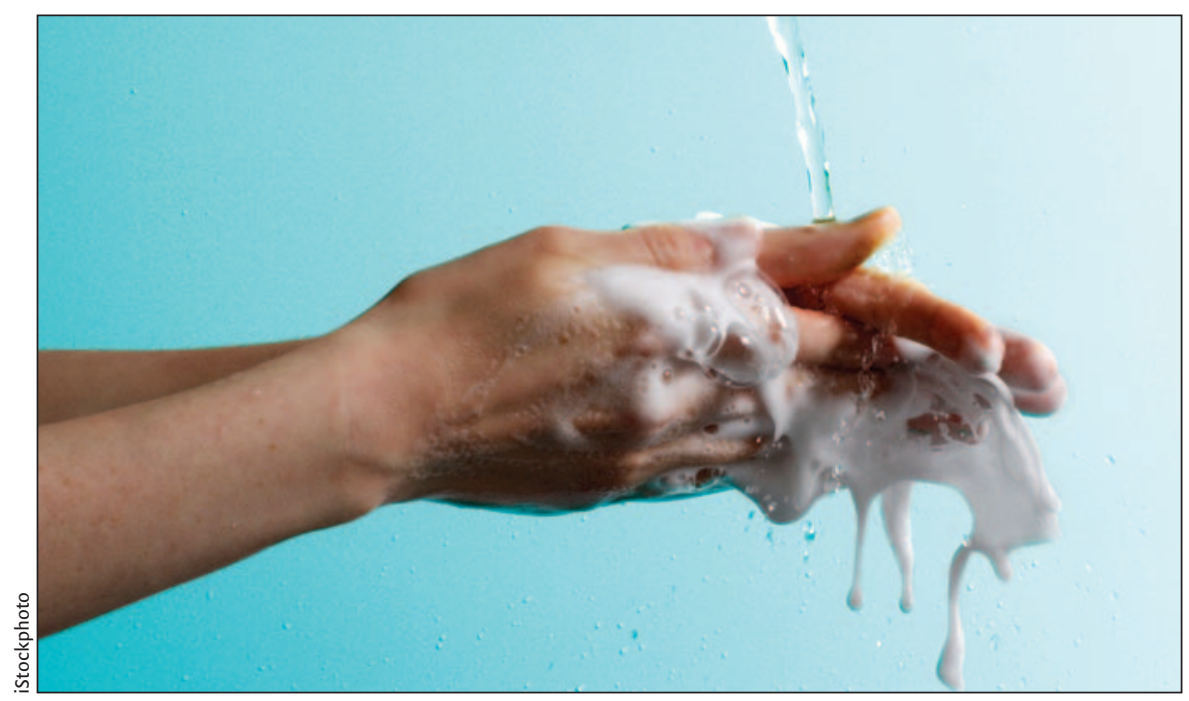

Washing hands with soap and water after using the toilet, before eating and before preparing food will decrease the incidence of diarrheal illness. If water is not accessible, an alcohol-based gel hand sanitizer is recommended.

running water is not accessible. Hands should be washed before eating, before preparing food, after using the toilet, changing diapers or other similar exposure, and after playing with or feeding pets. Hand hygiene after contact with potentially infected fomites or infected people is also appropriate. Consistent environmental cleaning with detergent, especially of frequently touched objects, is also recommended. Respiratory hygiene, or respiratory etiquette, is another current message directed to potentially infected people. It includes turning away and covering the mouth or nose when coughing or sneezing, together with appropriate disposal of tissues, hand hygiene, and staying home when ill with a cough and fever.

\section{Does hygiene work?}

Studies in developing countries evaluating interventions to promote personal and domestic hygiene in addition to safe water and food practices have documented improved health outcomes with improved hygiene practices. In a meta-analysis of comparative studies, the relative risk of diarrheal illness was 0.56 ( $95 \%$ confidence interval [CI] $0.33^{-0.93) ~ w i t h ~ h a n d w a s h i n g ~ i n-~}$ terventions and 0.72 (95\% CI $0.63-$ 0.83 ) with an education intervention. ${ }^{1}$

A randomized nonblinded trial in the United States evaluated a multifac- 
washing with soap in preschools and homes, the intervention significantly improved handwashing but had no impact on overall absenteeism or illnessrelated absenteeism. ${ }^{4}$

The effectiveness of recommended hygiene measures during an outbreak is not known.

\section{What are the harms of promoting hygiene?}

Some immediate concerns are skin irritation from the use of specific cleaning agents or damage to the skin barrier from excessive drying after repeated handwashing. Such effects on the skin may increase adherence of noncommensal flora and limit compliance. They are of particular concern in a dry environment, such as most of Canada in the winter.

Exposure to some irritative cleaning agents, including bleach, is occasionally associated with an increased occurrence of asthma-type symptoms or chronic bronchitis. More controversial is the "hygiene hypothesis," which suggests that the elevated rates of asthma and other atopic diseases in children in developed countries are attributable to decreased exposure to microbiologic antigens at an early age (i.e., too much cleanliness). This lack of exposure results in the immune system developing along a pathway that makes the child more susceptible to allergic illness.

A variation on the hygiene message, promoted by industry, is to recommend increased use of disinfectants such as triclosan for household or hand cleaning. There are, however, concerns that widespread use of antibacterial agents in routine household cleaning will promote bacterial resistance in the community. ${ }^{5}$

\section{What should patients be told?}

The evidence from studies in less developed countries is compelling: the adherence to usual recommendations for hand hygiene, including washing hands with soap and water after using the toilet, before eating and before preparing food, will decrease the incidence of diarrheal illness. Thus, standard recommendations for handwashing can be enthusiastically promoted. Accompanying the hand-hygiene message, however, must be recommendations for strategies to limit skin damage, in particular the consistent use of lotions to maintain skin integrity. The use of antibacterial soaps on a routine basis in the home or the community cannot currently be recommended. In settings where running water is not accessible, handwashing can be facilitated by the use of alcohol-based gel hand sanitizers. Standard kitchen practices for safe food preparation, including hand hygiene and environmental cleaning, should be promoted. Routine environmental cleaning is an important prac- tice, but there is no evidence to support consistent use of antibacterial products for household cleaning. Whether increased personal or environmental hygiene in the home will limit illness when an outbreak of diarrheal or respiratory infection occurs in the community is not known. Respiratory hygiene seems self-evident - none of us wants people coughing into our face - but the efficacy of these practices has not yet been critically assessed.

\section{Lindsay Nicolle \\ Department of Medicine \\ University of Manitoba \\ Winnipeg, Man.}

This article has been peer reviewed.

Competing interests: None declared.

\section{REFERENCES}

I. Fewtrell L, Kaufman RB, Kay D, et al. Water, sanitation, and hygiene interventions to reduce diarrhoea in less developed countries: a systematic review and meta-analysis. Lancet Infect Dis 2005;5: 42-52.

2. Sandora TJ, Taveras EM, Shih MC, et al. A randomized, controlled trial of a multifaceted intervention including alcohol-based hand sanitizer and handhygiene education to reduce illness transmission in the home. Pediatrics 2005; II6:587-94.

3. Larson EL, Lin SX, Gomez-Pichardo C, et al. Effect of antibacterial home cleaning and handwashing products on infectious disease symptoms: a randomized, double-blind trial. Ann Intern Med 2004; I40:32I-9.

4. Rosen L, Manor O, Engelhard D, et al. Can a handwashing intervention make a difference? Results from a randomized controlled trial in Jerusalem preschools. Prev Med 2006;42:27-32.

5. Aiello A, Larson E. Antibacterial cleaning and hygiene products as an emerging risk factor for antibiotic resistance in the community. Lancet Infect Dis 2003;3:50I-6.

\section{TRADITION}

Since I9II, CMAJ has been committed to advancing the science and art of medicine and promoting public health. 